PROCEEDINGS OF THE

AMERICAN MATHEMATICAL SOCIETY

Volume 131, Number 1, Pages 231-233

S 0002-9939(02)06756-4

Article electronically published on June 27, 2002

\title{
A REMARK ON REAL COBOUNDARY COCYCLES IN $L^{\infty}$-SPACE
}

\author{
RYOTARO SATO
}

(Communicated by Michael Handel)

\begin{abstract}
Let $T$ be an ergodic automorphism of a probability measure space $(\Omega, \mathcal{A}, m)$ and let $f$ be a real-valued measurable function on $\Omega$. We deduce a necessary and sufficient condition for the existence of $L^{\infty}$-solutions of the cohomology equation $f=h \circ T-h$, by using the recent result of Alonso, Hong and Obaya.
\end{abstract}

Let $(\Omega, \mathcal{A}, m)$ be a probability measure space and let $T$ be an ergodic automorphism of $(\Omega, \mathcal{A}, m)$. Every real-valued measurable function $f$ on $\Omega$ defines an additive real $T$-cocycle

$$
S_{n} f=\sum_{j=0}^{n-1} f \circ T^{j}, \quad n>0 .
$$

Recently Alonso, Hong and Obaya proved in [1] that a necessary and sufficient condition for the existence of $L^{p}$-solutions of the cohomology equation $f=h \circ T-h$, with $0<p<\infty$, is that $f \in L^{p}(\Omega, m)$ and there exists a measurable subset $A \subset \Omega$ with $m(A)>0$ and an increasing sequence $\left\{n_{k}\right\}$ of positive integers with

$$
\sup _{k \geq 1} \frac{1}{n_{k}} \sum_{j=1}^{n_{k}} \int_{A}\left|S_{j} f\right|^{p} d m<\infty .
$$

For related results we refer the reader to [2] and 3]. In this note we would like to consider the case $p=\infty$ and prove the following theorem.

Theorem. Let $f \in L^{p}(\Omega, m)$ for some $p$ with $0<p \leq \infty$. Then there exists a function $h$ in $L^{\infty}(\Omega, m)$ with $f=h \circ T-h$ if and only if there exists a measurable subset $A \subset \Omega$ with $m(A)>0$ and an increasing sequence $\left\{n_{k}\right\}$ of positive integers with

$$
\sup _{k \geq 1} \frac{1}{n_{k}} \sum_{j=1}^{n_{k}}\left\|S_{j} f \cdot \chi_{A}\right\|_{\infty}<\infty
$$

Proof. Suppose $f=h \circ T-h$ for some $h \in L^{\infty}(\Omega, m)$. Then, since $S_{j} f=h \circ T^{j}-h$ for $j \geq 1$, we have $\left\|S_{j} f\right\|_{\infty} \leq 2\|h\|_{\infty}$, whence (3) holds for $A=\Omega$ and $n_{k}=k$.

Received by the editors August 31, 2001.

2000 Mathematics Subject Classification. Primary 37A20, 28D05, 47A35.

Key words and phrases. Ergodic automorphism, additive real cocycle, cohomology equation, coboundary. 
Conversely, suppose the second condition of the Theorem holds. Since $0<r<p$ implies $L^{p}(\Omega, m) \subset L^{r}(\Omega, m)$, we may assume from the beginning that $0<p \leq 1$. Then we have

which implies

$$
\int_{A}\left|S_{j} f\right|^{p} d m \leq 1+\left\|S_{j} f \cdot \chi_{A}\right\|_{\infty} \quad \text { for } j \geq 1
$$

$$
\sup _{k \geq 1} \frac{1}{n_{k}} \sum_{j=1}^{n_{k}} \int_{A}\left|S_{j} f\right|^{p} d m<\infty .
$$

Thus, by Theorem 3.2 of [1], there exists a function $h$ in $L^{p}(\Omega, m)$ with $f=h \circ T-h$. It suffices to show that $h$ is a function in $L^{\infty}(\Omega, m)$.

To do so, we notice that there exists a positive number $\delta$ with

$$
m(A \cap\{\omega:|h(\omega)| \leq \delta\})>0 .
$$

Therefore, we may assume from the beginning that

$$
|h| \leq \delta \quad \text { on } \quad A \text {. }
$$

Then, using the relations

$$
\left\|h \circ T^{j} \cdot \chi_{A}\right\|_{\infty}=\left\|\left(S_{j} f+h\right) \cdot \chi_{A}\right\|_{\infty} \leq\left\|S_{j} f \cdot \chi_{A}\right\|_{\infty}+\delta,
$$

we see from (3) that

$$
M:=\sup _{k \geq 1} \frac{1}{n_{k}} \sum_{j=1}^{n_{k}}\left\|h \circ T^{j} \cdot \chi_{A}\right\|_{\infty}<\infty .
$$

By this inequality, we will prove below that $h \in L^{\infty}(\Omega, m)$.

For this purpose, let $\alpha$ be a positive number such that there exists a measurable subset $B \subset \Omega$ with

$$
m(B)>0 \text { and }|h| \geq \alpha \text { on } B .
$$

Since $T$ is an ergodic automorphism by hypothesis, the Birkhoff Ergodic Theorem implies that

$$
\lim _{k \rightarrow \infty} \frac{1}{n_{k}} \sum_{j=1}^{n_{k}} \chi_{A}\left(T^{-j} \omega\right)=m(A)>0
$$

for almost all $\omega \in \Omega$. Hence, we can choose a measurable subset $B_{1} \subset B$ with $m\left(B_{1}\right)>0$ and a positive integer $K$ with

$$
\frac{1}{n_{K}} \sum_{j=1}^{n_{K}} \chi_{A}\left(T^{-j} \omega\right)>2^{-1} m(A) \quad \text { for all } \omega \in B_{1} .
$$

Thus, taking into account (5) and (6), we get

$$
\begin{aligned}
\infty>M & \geq \frac{1}{n_{K}} \sum_{j=1}^{n_{K}}\left\|h \circ T^{j} \cdot \chi_{A}\right\|_{\infty}=\frac{1}{n_{K}} \sum_{j=1}^{n_{K}}\left\|h \cdot \chi_{A} \circ T^{-j}\right\|_{\infty} \\
& \geq \frac{1}{n_{K}} \sum_{j=1}^{n_{K}}\left\|\alpha \chi_{B} \cdot\left(\chi_{A} \circ T^{-j}\right)\right\|_{\infty} \geq \alpha \cdot\left\|\chi_{B_{1}} \cdot\left(\frac{1}{n_{K}} \sum_{j=1}^{n_{K}} \chi_{A} \circ T^{-j}\right)\right\|_{\infty} \\
& \geq \alpha \cdot 2^{-1} m(A) .
\end{aligned}
$$

This shows that $\alpha$ cannot be so large, and thus $h \in L^{\infty}(\Omega, m)$ must follow. This completes the proof. 


\section{REFERENCES}

[1] A. I. Alonso, J. Hong and R. Obaya, Absolutely continuous dynamics and real coboundary cocycles in $L^{p}$-spaces, $0<p<\infty$, Studia Math. 138 (2000), 121-134. MR 2001i:37010

[2] I. Assani, A note on the equation $Y=(I-T) X$ in $L^{1}$, Illinois J. Math. 43 (1999), 540-541. MR 2000j:47016

[3] M. Lin and R. Sine, Ergodic theory and the functional equation $(I-T) x=y$, J. Operator Theory 10 (1983), 153-166. MR 84m:47015

Department of Mathematics, Okayama University, Okayama, 700-8530 Japan

E-mail address: satoryot@math.okayama-u.ac.jp 